\title{
LISA optical metrology: tilt-to- pathlength coupling effects on the picometer scale
}

Nils F. Hasselmann, Christina Brugger, Tim Vogel, Ewan Fitzsimsons, Ulrich Johann, et al.

Nils F. Hasselmann, Christina Brugger, Tim Vogel, Ewan D. Fitzsimsons, Ulrich Johann, Gerhard Heinzel, Dennis Weise, Alexander Sell, "LISA optical metrology: tilt-to-pathlength coupling effects on the picometer scale," Proc. SPIE 11852, International Conference on Space Optics - ICSO 2020, 1185242 (11 June 2021); doi: 10.1117/12.2599636

SPIE Event: International Conference on Space Optics - ICSO 2021, 2021, Online Only 


\section{International Conference on Space Optics-ICSO 2020}

Virtual Conference

30 March-2 April 2021

Edited by Bruno Cugny, Zoran Sodnik, and Nikos Karafolas
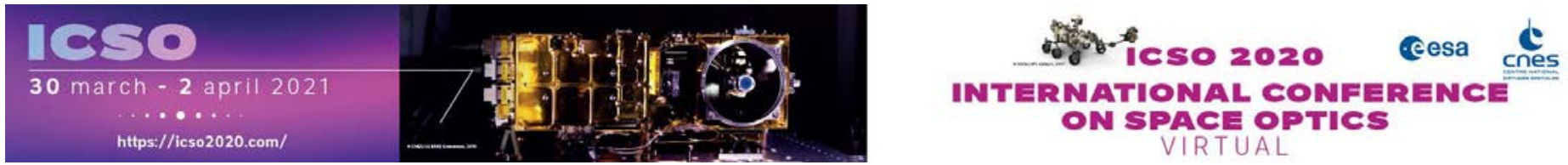

\section{LISA optical metrology: tilt-to-pathlength coupling effects on the picometer scale}

\section{- esa issopocestings denes}




\title{
LISA optical metrology: Tilt-to-pathlength coupling effects on the picometer scale
}

\author{
Nils F. Hasselmann ${ }^{\mathrm{a}}$, Christina Brugger ${ }^{\mathrm{a}}$, Tim Vogel ${ }^{\mathrm{a}}$, Ewan D. Fitzsimsons ${ }^{\mathrm{b}}$, Ulrich Johann ${ }^{\mathrm{a}}$, \\ Gerhard Heinzel ${ }^{\mathrm{c}}$, Dennis Weise ${ }^{\mathrm{a}}$, and Alexander Sell ${ }^{\mathrm{a}}$ \\ ${ }^{a}$ Airbus Defence and Space GmbH, Claude-Dornier-Straße, Immenstaad, Germany \\ ${ }^{\mathrm{b}}$ UK Astronomy Technology Centre, Royal Observatory Edinburgh, Edinburgh, UK \\ ${ }^{\mathrm{c}}$ AEI, Callinstraße 38, Hannover, Germany
}

\begin{abstract}
Airbus Defence and Space GmbH currently investigates a number of promising payload concepts for interferometric space-bourne gravitational wave observatories; focusing on beam pointing for the laser links. ${ }^{1}$ To this end, one of the setups implements "In-Field Pointing (IFP)", which is a payload design employing the actuation of a tiltable mirror positioned in an intermediate pupil plane of a wide field off-axis telescope. Here, the lateral alignment of actuated mirror and relay stages imaging the intermediate pupil to the detector plane using tilt-to-length coupling effects will be discussed.
\end{abstract}

\section{INTRODUCTION}

The LISA mission sets out to observe gravitational waves from space in a measurement band from $0.1 \mathrm{mHz}$ to 0.1 $\mathrm{Hz}$, which is so far not accessible to ground-based detectors. LISA consists of a triangular formation of satellites separated by $2.5 \mathrm{Gm}$ that will be trailing earth on a heliocentric orbit. ${ }^{2}$ Annual orbital dynamics vary the angles of the triangle, necessitating an actuated field of view of the telescopes for the links, while maintaining $\mathrm{pm} / \sqrt{\mathrm{Hz}}$ noise levels in the interferometric readout. Coupling of angular jitter of the spacecraft and pointing mechanisms to the measured piston ("TTL" i.e. "tilt-to-length") due to angular gradients of the optical pathlength is among the key contributors to the overall measurement noise, limiting the allowable angular noise to a $10 \mathrm{nrad} / \sqrt{\mathrm{Hz}}$ level ${ }^{3 *}$.

In comparison to the baseline architecture "Telescope Pointing", only small components are actuated, spacecraft internal phase referencing is simplified, and a single inertial reference sensor is sufficient for operating the system. ${ }^{4}$ The prototype mechanism ${ }^{5}$ also serves as example for various mechanisms proposed for different LISA payload architectures, which might employ similar actuator technology.

On-ground tests are challenging, both due to the required angular-jitter and due to additional environmental perturbations. We will present the first TTL measurements performed using a setup featuring a representative telescope, interferometer and pointing mechanism during alignment as well as the alignment strategy based on numerical and analytical models of TTL effects. Additionally, angular jitter noise measurements will be shown and the obtained wavefront error for the telescope from which far-field TTL coupling is computed.

\section{EXPERIMENTAL SETUP}

The setup, as sketched in Figure 1, features an off-axis wide field reflective all Zerodur telescope with a 5x magnification followed by a $5 x$ refractive stage in Kepler configuration, yielding a total magnification of 25 . The Entrance pupil diameter is $150 \mathrm{~mm}$ with a Gaussian measurement beam of a $25 \mathrm{~mm}$ radius. In this pupil, a plane mirror is positioned, reflecting back the incoming light. This mirror can be replaced by an actuated one (also referred to as "Space-Craft-Simulator" or simply "SCS") in order to simulate the motion of the distant spacecraft and thus allow TTL measurements over the whole field of view. In the exit pupil of the reflective stage, which

Further author information: A.A.S.: E-mail: alexander.a.sell@airbus.com

*If the angular dependency $s(\phi, \eta)$ of the optical path-length is linearized around a nominal angle $\left(\phi_{0}, \eta_{0}\right)$, an angular jitter $(\delta \phi, \delta \eta)$ will consequently cause an apparent path-length jitter $\left.\delta s=\nabla_{(} \phi, \eta\right)\left.s\right|_{\left(\phi_{0}, \eta_{0}\right)} \cdot(\delta \phi, \delta \eta)^{T}$. 
is ideally coincident with the entry pupil of the refractive stage and also referred to as intermediate pupil of the system, the actuated mirror for beam steering is positioned (also "In-Field-Poining mirror" or "IFPM"). This mirror is responsible for compensating the tilt introduced by the SCS. Following the telescope is the interferometer ("IFO") bench containing all infrastructure required for routing of local oscillator and measurement beams and the phase detection using various quadrant photo diodes ("QPDs") and single element diodes. Knowledge of the detailed make up of the IFO bench is not required for the purposes of this and hence not discussed here. However, one should note, that a unity magnification refractive relay stage is positioned behind the beamsplitter overlaying local oscillator and measurement beam in order to complete pupil imaging of the entry pupil all the way to the detector plane.

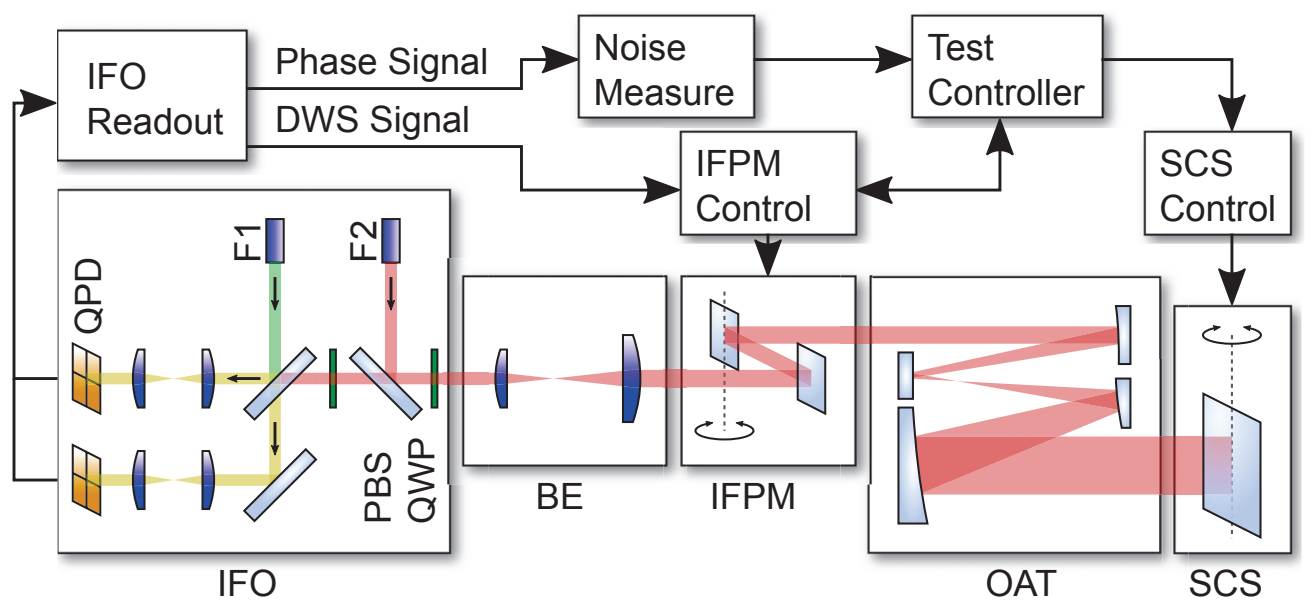

Figure 1: Schematic of the experimental setup. SCS: Space-Craft-Simulator, OAT: Off-Axis reflective telescope, IFPM: In-Field-Pointing actuated mirror, BE: Beam expander, QWP: Quarter-wave plate, PBS: Polarizing beam splitter, F1/2: Fiber launchers 1/2, QPD: Quadrant photodiode, IFO: Interferometer (bench).

\section{NUMERICAL COMPUTATION OF IFP TELESCOPE TTL}

By means of ray tracing, the relationship of the optical pathlength and scan angles of the actuated mirrors can be computed. Ideal surfaces and alignment are assumed and the optical pathlength is computed solely by using ray optics.

The ray tracer is a useful tool to perform sensitivity studies in order to investigate the effects of misalignments or combinations thereof. During alignment, integration and testing, it can be used to derive the deviation of mirror positions from their ideal and provide us with information on how to place them in order to rectify these. In Figure 2, the optical pathlength difference in dependence of the scan angle of the IFPM mirror is shown for the complete IFP telescope. This Data represents the case of a double pass through the setup with the SCS mirror being tilted by $\frac{2 \varphi}{5}$, in order to compensate for a continuous variation of the mechanical angle $\varphi$ of the IFPM. In this mode of operation, the telescope TTL might be determined. Next to this plot, the derivative of the optical pathlength (OPD) is shown representing the TTL coupling factor for each scan angle. Mirror motion due to parasitic deformations of the flexure hinges used in IFPM and SCS is not accounted for. Such effects will be discussed in section 4.1 .

The coupling factor for jitter in the angular direction orthogonal to the scan direction was computed to yield pathlength noise contributions below $0.02 \mathrm{pm} / \sqrt{\mathrm{Hz}}$ over the field of view of the telescope. Assuming a total pointing jitter of $10 \mathrm{nrad} / \sqrt{\mathrm{Hz}}$ in the entrance pupil, TTL will contribute to pathlength noise with less than $3.5 \mathrm{pm} / \sqrt{\mathrm{Hz}}$ during operation according to this model. Before performing actual measurements in our experimental setup, IFPM mirror axis and refractive stages have to be aligned. 

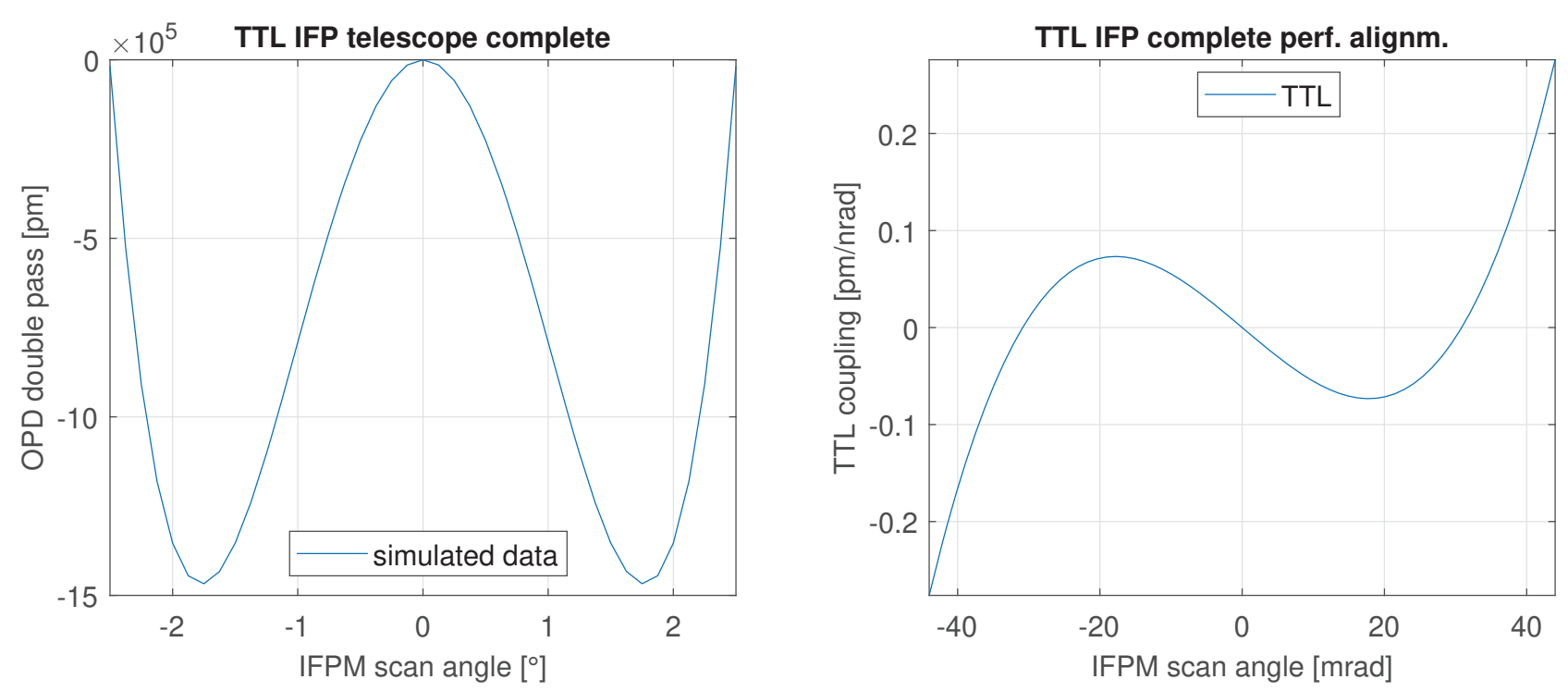

Figure 2: The optical pathlength and its derivative in dependence of the IFPM scan angle. IFPM and SCS are scanned such that the mechanical angle of SCS is $\frac{2 \varphi}{5}$, with $\varphi$ being the mech. angle of the IFPM.

\section{LATERAL ALIGNMENT OF THE ACTUATED MIRROR AND REFRACTIVE RELAY STAGES}

The following considerations are valid for incomplete pupil imaging with no refractive relay stage (BE), a double pass through the system and the SCS not compensating tilt of the IFPM. In such case, the change in optical pathlength with respect to the value at mechanical angle $\varphi=0$ in dependence of a parametric lateral misalignment and the mechanical angle $\varphi$ can be derived analytically. The parameter $L$ defines the distance, the light has to travel from the intermediate pupil plane to the detector plane without passing through an optical relay system. The lateral offset of the rotational axis of the mirror tilted in the intermediate pupil plane is named $\Delta x$. This (vertical) lateral offset is defined in the direction perpendicular both, to the nominal rotational axis of hinge and to the surface normal of the untilted mirror. As a lateral offset in direction of the rotational axis (horizontal) does not contribute to TTL all mentioning of lateral offsets, unless otherwise stated, refers to vertical lateral offsets. The cosine factor is accounting for the reduced piston due to the angle of incidence with respect to the IFPM mirror normal being varied around an angle of $\Phi_{0}=22.5^{\circ}$ in nominal IFPM scan direction. The dependence of optical pathlength difference $s$ on mechanical angle is:

$$
s(-\varphi)=\left(\frac{L}{\cos (-4 \varphi)}-L+2 \Delta x\left(\sin (-\varphi)\left(\cos \left(\Phi_{0}-\varphi\right)+\cos \left(\Phi_{0}+3 \varphi\right)\right)\right)\right)
$$

For small angles $\varphi$, Taylor expansion of this expression yields

$$
s(-\varphi) \approx \frac{L}{2}(4 \varphi)^{2}-2 \Delta x\left(0.92388 \cdot 2 \varphi+0.388 \cdot 2 \varphi^{2}\right)
$$

Substituting $\tilde{\varphi}=4 \varphi$ where $\tilde{\varphi}$ is the angle of the ray returning against the incoming ray and thus the angle measured by means of DWS by the QPDs/detector gives us

$$
s(\tilde{\varphi}) \approx \frac{L}{2}\left(\tilde{\varphi}^{2} \cdot\left(1-\frac{0.388 \Delta x}{2 L}\right)-\frac{2 \Delta x \cdot 0.92388}{L} \tilde{\varphi}\right)
$$

A second approximation can be used in order to further simplify the expressions as $\left(1-\frac{0.388 \Delta x}{2 L}\right) \approx 1$ due to $\frac{\Delta x}{L}<<1$. This step is not required, but makes the final result much more compact and intuitive. Introducing a 
parameter $a:=\frac{0.92388 \cdot 2 \Delta x}{L}$ and rewriting the equation yields

$$
s(\tilde{\varphi}) \approx \frac{L}{2}\left(\tilde{\varphi}-\frac{a}{2}\right)^{2}-\frac{a^{2}}{4}
$$

The location of the optical pathlength minimum for $L=1.1993 \mathrm{~m}$ and for a typical misalignment of $\Delta x=30 \mu \mathrm{m}$ is expected at a DWS angle of

$$
\tilde{\varphi} \approx \frac{a}{2}=23110 \mathrm{nrad}
$$

The value obtained by this very simple model is in agreement with the value derived by the ray tracer $(\tilde{\varphi}=$ $23070 \pm 50 \mathrm{nrad}$ ) in case one introduces the same misalignment and removes the refractive relay stages from the setup used earlier in the computation of data shown in Figure 2.

This shift in minimum position can be minimized by successively translating the IFPM mirror using the micrometer screws of the mounts. In doing so, one can home in on the ideal position. Limitations arise due to the error of the fit to the data and data quality (especially for measurements not taken in vaccum) and thus play a role in determination of the accuracy the lateral misalignment can be measured with (typically around $\pm 15 \mu m$ ). Simulations performed using the ray tracer as well as experimental data recorded during lateral alignment of the rotational axis of the IFPM with respect to the nominal position given by IFO bench alignment is shown in Figure 3. Plotted is the optical pathlength difference versus the optical angle in scan direction (as determined by differential wavefront sensing (DWS) for the experimental data (right)).
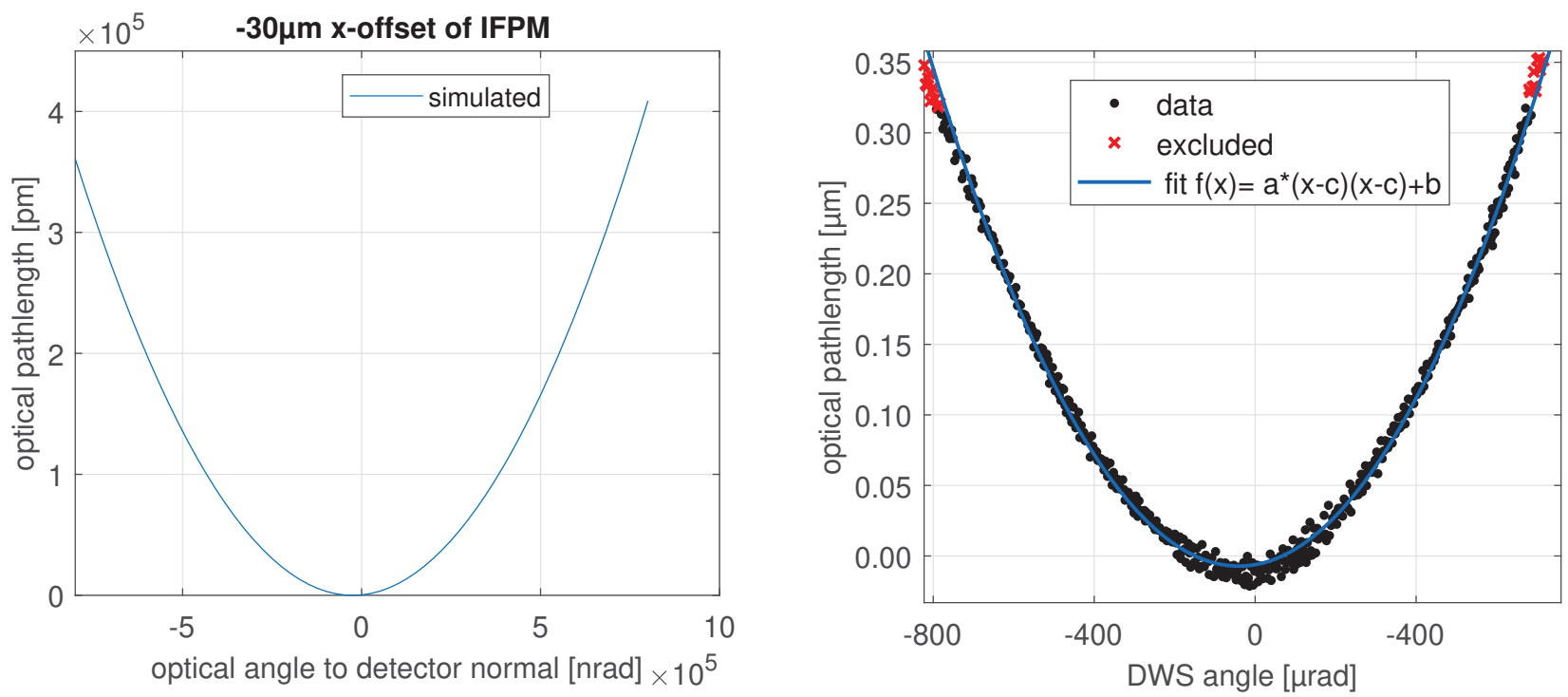

Figure 3: The parametric dependence of the shift of the minimum position from the IFPM rotational axis lateral misalignment can be used for alignment. On the left, the simulated optical pathlength is plotted against the optical angle. On the right, experimental data is fitted to the analytical model.

After alignment is done to the level, where measurement errors do not allow for a detection of a difference to the nominal position, the $5 \mathrm{x}$ magnification refractive stage can be added. Before the beam expander is positioned within the setup, the lenses are prealigned using a Shack-Hartman sensor for wavefront error measurement in order to obtain the minimal wavefront error. A linear stage is used to position a mirror of negligible wavefront error in between the empty space the refractive stage will be placed in and the IFPM. Alignment of that mirror is done such to reflect the light back in itself (DWS angle $=0$ ). A pick-off mirror is placed in the interferometer in order to route a fraction of the measurement beam into the Shack-Hartmann sensor. In doing so, one can 
optimize spot position and DWS signal on a QPD, while also optimizing the wavefront error of only the refractive stage or refractive and reflective stages combined. First, the refractive stage as a whole is manually positioned before using the micrometer screws of the lens mounts for fine positioning. The side conditions to be obeyed during this are that the DWS angle and the spot position are not changed with respect to the original values. After this process, the mirror mounted to the linear stage is moved to the side, allowing for the detection of the combined wavefront error of refractive and reflective stages. This data is relevant for far field TTL and will be discussed later. After lateral placement with respect to the nominal position is complete, the lateral beam alignment with respect to the rotational axis of the IFPM can be done in analogy to before. Performing these adjustments is done while screening the wavefront error.

If one seeks to minimize TTL due to IFPM mirror piston this way (compensating a misalignment of the IFPM rotational axis by lateral translation of the refractive stage), one should be aware that also a re-positioning of the actuated plane mirror simulating the distant spacecraft is in order; otherwise the shifted beam just generates the (equal) TTL at the plane mirror instead at the IFPM. As determination of the change in optical pathlength over the entire field of view demands making use of the actuated plane mirror due to the limited field of view of the IFO $(\approx 1 \mathrm{mrad})$, the static mirror initially used must be replaced. Furthermore, the pupil imaging must be completed by integration of the last relay stage ${ }^{\dagger}$. When placing the SCS in the entry pupil, longitudinal alignment can be done using a laser tracker or similar metrology device, not being critical as a result of the 625 times longitudinal magnification of the telescope. Tilt of the SCS mirror is adjusted using the DWS signal as feedback. A misalignment along the rotational axis is of no concern, as it is irrelevant to mirror piston and can be done using a secondary laser tracker. Placement in vertical lateral direction orthogonal to mirror rotational axis and surface normal of the mirror is done such, that TTL effects due to lateral misalignments of the IFPM are compensated. This allows for measurement of telescope TTL plus TTL contributions from parasitic mirror motion of both actuated mirrors by scanning both mirrors simultaneously with SCS mechanical angle being equal to $\frac{2 \varphi}{5}$, accommodating the magnification of the reflective stage.
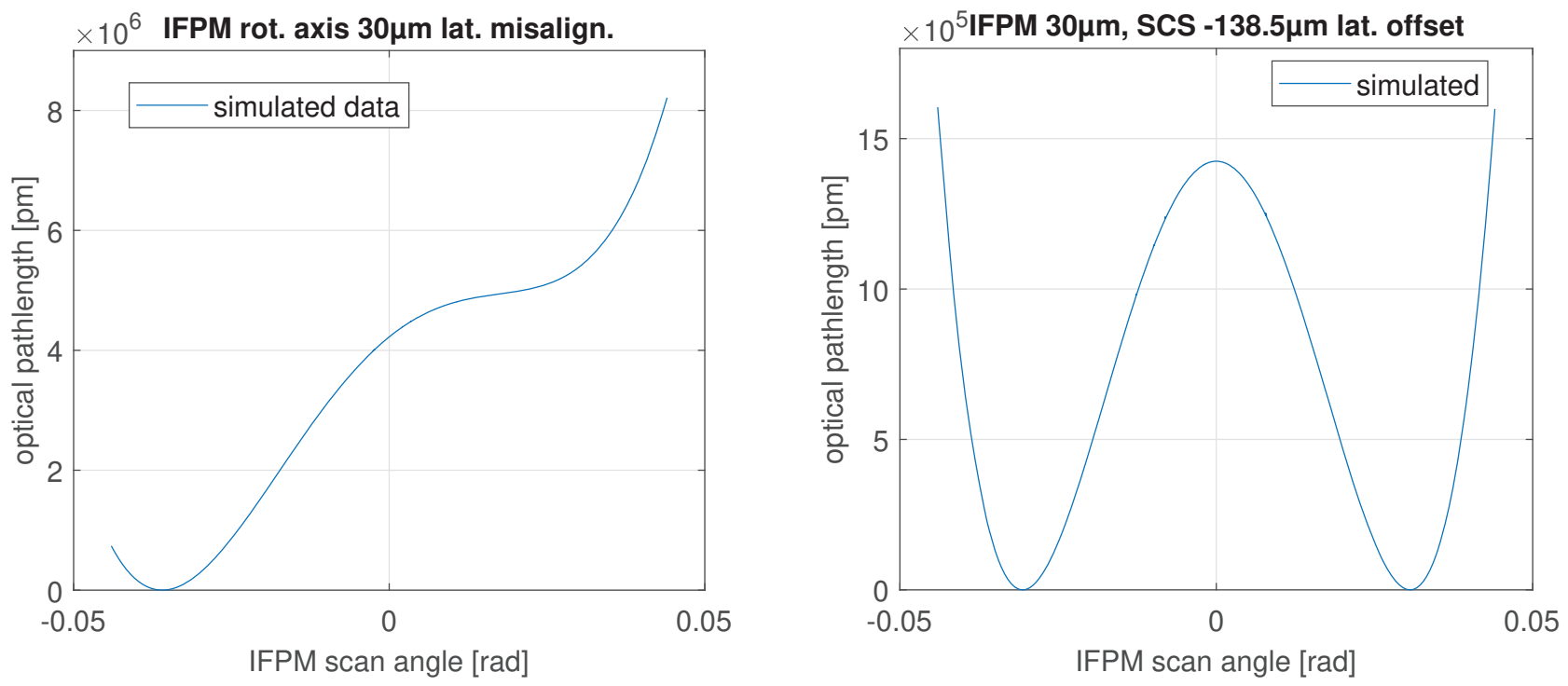

Figure 4: For the measurement of the telescope properties, additional vertical misalignments can be introduced in order to compensate undesired effects.

In Figure 4, the optical path difference for misaligned IFPM and nominal positioned SCS mirror in the entry pupil is shown on the left and the case with the SCS misaligned to compensate TTL due to a lateral misalignment

${ }^{\dagger}$ The unity magnification refractive stage is aligned using spot position and DWS data as well as wavefront error measurements. 
of the IFPM is shown on the right. Even with all pupil imaging optics in place, we still are able to extract the lateral misalignment of the IFPM rotational axis by scanning the IFPM around (DWS) angle zero while not tilting the SCS with respect to its nominal angular position: In case one images the pupil plane onto the detector the value of the geometrical lever arm $L$ becomes very small. As a matter of fact $L$ can be expected to be not greater than a factor of 10 compared to $\Delta x$. With equation 2 it follows, that TTL due to this longitudinal misalignment is suppressed and hence can be neglected. Therefore we obtain (for the same typical values as before):

$$
\frac{\partial s(\varphi)}{\partial \varphi} \approx 0.92388 \cdot 4 \Delta x=0.11086 \mathrm{pm} \mathrm{nrad}^{-1}
$$

Which is again in agreement with the data obtained using the ray tracer $\left(\frac{\partial s(\varphi)}{\partial \varphi}=0.11086 \pm 0.00002 \mathrm{pm} \mathrm{nrad}^{-1}\right)$. In Figure 5 one can see the OPD due to lateral misalignment of the IFPM rotational axis with respect to spot

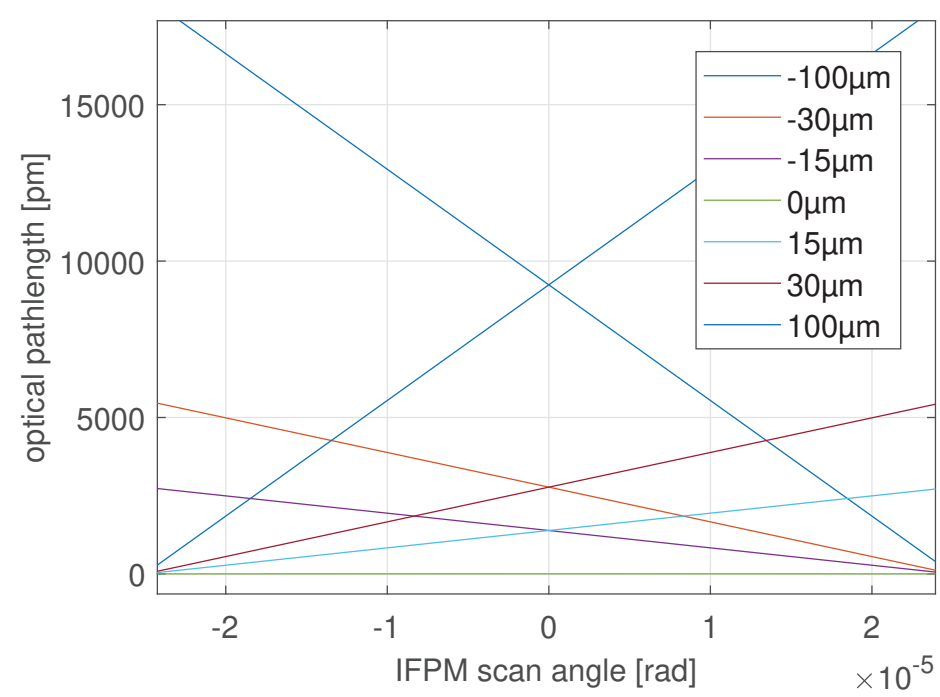

Figure 5: Ray tracing simulations of optical pathlength over scan angle when scanning the IFPM and while the SCS is maintaining a total angle of zero in case of complete pupil imaging. An offset corresponding to the minimal value was subtracted from each linear trend.

position over the IFO field of view for various lateral misalignments as computed by the raytracer.

As predicted by the analytical model, we obtain an almost linear slope when scanning the IFP mirror around $\varphi=0$ while keeping the SCS mirror static. The uncertainty of the slope will be dominating the measurement error of the lateral position of the rotational axis compared to the true one and thus define how well the IFPM could be positioned using this method and what residual mirror piston and associated TTL is to be expected from using this technique on its own.

Longitudinal misalignments of QPD to pupil plane appear as an additional quadratic contribution and can be determined by accommodation of this fact in the model fitted to the data set. This information can be used to correctly position the QPD in longitudinal direction.

\subsection{Parasitic mirror motion due to hinge deformation}

Based on an analytical model of telescope OPD taking into account hinge-deformation-driven optical piston of the two actuated mirrors used in the measurement scheme; we were able to implement a simple algorithm reconstructing the optical (and thus mechanical) piston of the IFPM mirror by scanning the IFPM mirror 

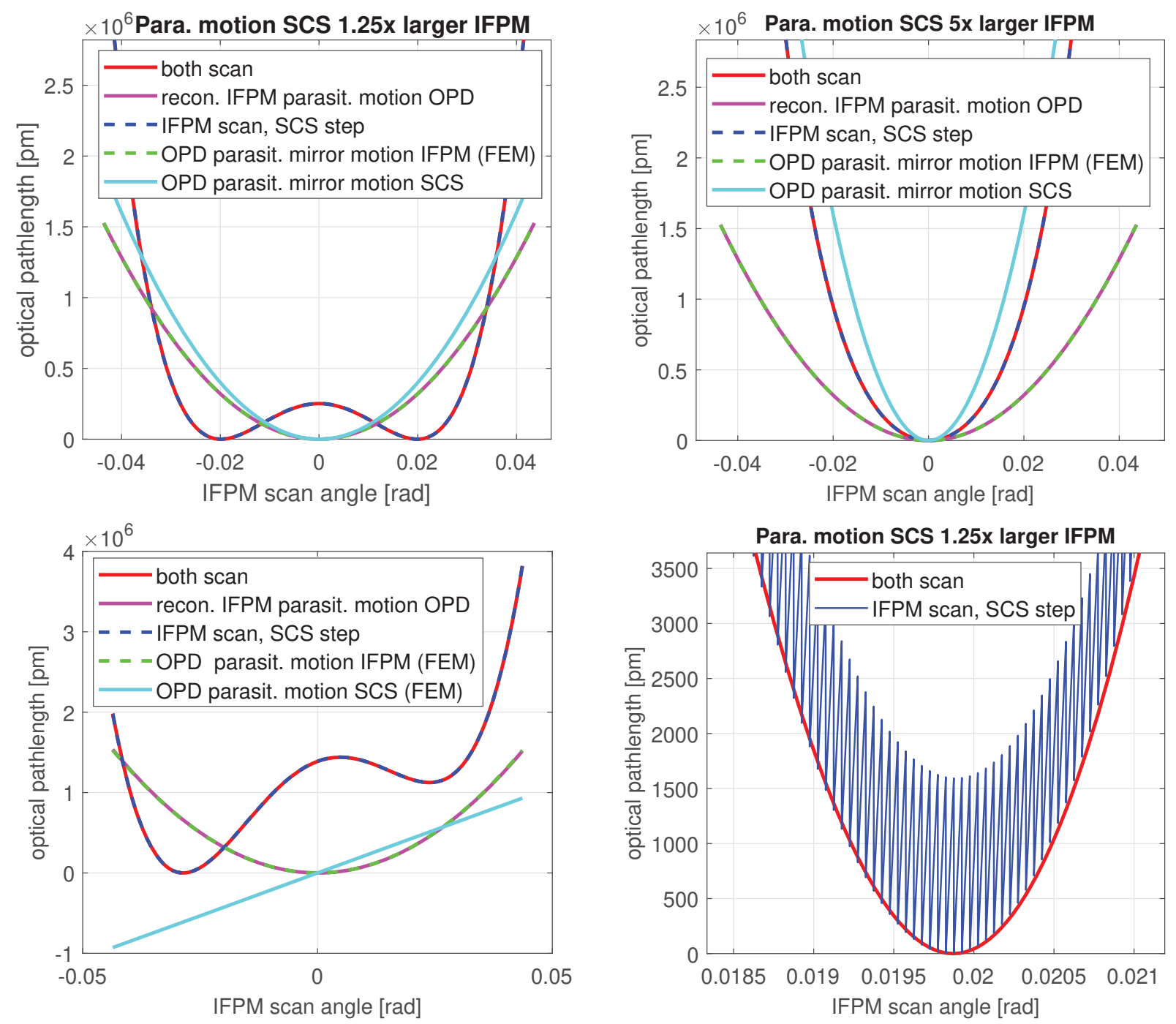

Figure 6: Optical pathlength (OPD) dependence on IFPM scan angle in case both actuators are scanned continuously (red). Also plotted are: The reconstructed OPD due to parasitic motion of the the IFPM mirror (magenta), the curve resulting from scanning the IFPM while repositioning the SCS step wise (blue) as well as the OPD due to parasitic mirror motions for IFPM (green) as predicted by means of FEM analysis, and for the SCS as scaled from the IFPM FEM analysis (light blue). The top right figure shows the very same curves for parasitic motion of the SCS that is five times larger than that of the IFPM. The lower left plot features the curves using dedicated FEM results for both mechanisms as inputs and the lower right plot shows the substructure from which the IFPM OPD can be reconstructed using the scan-step method (see text).

continuously while letting the SCS mirror perform a stepping motion to compensate beam steering of the IFPM in order to stay within the field-of-view of the IFO (s. Figure 6). The model approximates the optical pathlength caused by the telescope and the actuated mirrors each via polynomials. The scanned angle is defined as a superposition of saw tooth function and step function yielding a linear increase in angle, whereas the SCS-angle corresponds to the scaled steps alone. Our model shows, that a reconstruction of the IFPM piston can be simply achieved by removing jumps from the curve, where steps from the SCS occur, for example. Input data for optical piston created by parasitic hinge deformation was generated by means of FEM-calculations of the displacements, 
and an analytical model transferring these displacements into mirror/optical piston in case of the IFPM hinge (Fig. 6, green curve). Optical piston due to SCS hinge deformation was initially assumed to be a scaled version of the IFPM piston, later dedicated FEM simulations were performed (light blue curve, lower left).

Vice versa, the SCS mirror can be scanned and the IFPM mirror stepped. Such a measurement in combination with data from an additional measurement where both mirrors are continuously scanned such that the beam is reflected back in itself can also be used to reconstruct the contribution of the hinge deformation of the IFPM to OPD. However, the step-scan method is not sensitive to the piston generated by the SCS mirror. Thus, it can not be differentiated this way.

This requires one to perform additional characterization measurements or perform dedicated FEM simulations (considering any pre-tension present in the hinges of the mechanism) before AIT of the SCS into the setup (s. Figure 6 down,left). FEM simulations of the SCS however show, that the parasitic mirror motion mostly affects the longitudinal direction. The TTL due to parasitic hinge deformation in longitudinal direction can be compensated by introducing an additional lateral offset to the SCS hinge postion. In doing so, the TTL contribution of the SCS can become as small as $0.008 \mathrm{pm} / \sqrt{\mathrm{Hz}}$.

\section{EXPERIMENTAL FINDINGS}

This very brief section details experimental findings with respect to angular jitter noise as well as wavefront error of the IFP setup. While the jitter data in combination with the TTL coupling factors is key in apportioning the resulting path-length noise, the wavefront error determines the TTL-effect when sampling the expanded beam from the telescope remotely.

\subsection{Angular jitter noise of the setup}

Angular noise performance can be measured using differential wave sensing, i.e. spatially resolved sensing of the interference pattern. An amplitude spectral density for a typical measurement of the angle of the beam after a double pass through the telescope optics is shown in Figure 7. Apparently, the overall noise exceeds our set goal of $10 \mathrm{nrad} / \sqrt{\mathrm{Hz}}$, which we attribute to the mounting concept of our reflective telescope optics. These optics are supported isostatically via Zerodur spheres on their bottom, whose elasticity supports nick modes of the optics. Those modes are excited by seismic noise and give rise to the excess angular jitter observed. In order to be compliant with initially set goals, further improvements have to be implemented. These measures must yield up to one order of magnitude reduction in angular noise level. The reference path with its mirrors being directly bonded to the Zerodur baseplate, does exhibit angular noise compliant with the goal.

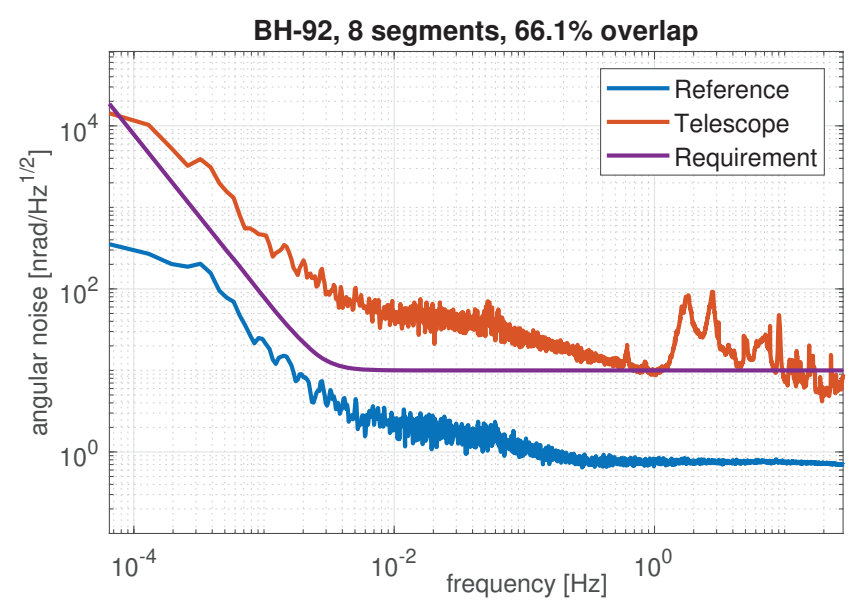

Figure 7: Angular jitter (in scan direction) of the optical beam after a double pass through the telescope optics (red curve). For comparison, the jitter of a monolithically bonded reference path is shown as well (blue curve). 


\subsection{Wavefront error and far-field TTL}

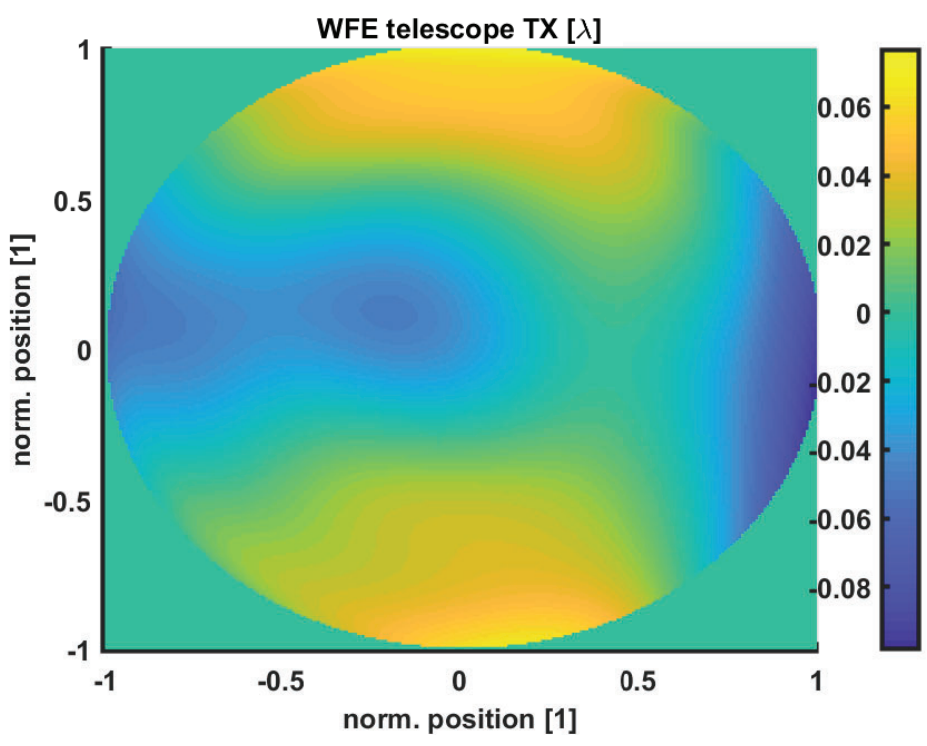

Figure 8: Combined wavefront error of the complete telescope (comprised of a $5 \mathrm{x}$ refractive and a $5 \mathrm{x}$ reflective stage). The RMS wavefront error amounts to $\approx 0.035 \lambda$.

The wavefront error(WFE) for a field angle of 0 was determined by means of a Shack-Hartman sensor (SHS). The experimentally determined WFE after decomposition into Zernicke polynominals can be seen in Figure 8. Upon decomposition into Zernicke polynominals (Coefficients of the lowest 36 Zernicke polynominals in Fringe indices notation are included), the data is transformed into a far-field phase by the Kirchhof integral using Fraunhofer approximation. As the SHS emits a collimated beam of $3.15 \mathrm{~mm}$ radius, the telescope radius in the computation of the far-field phase angular derivative was set to $25 \cdot 3.15 \mathrm{~mm}=78.75 \mathrm{~mm}$. Far-field TTL plotted versus the pointing angles $\phi$ and $\eta$ of the sending spacecraft is shown in Figure 9. Within a field of regard of
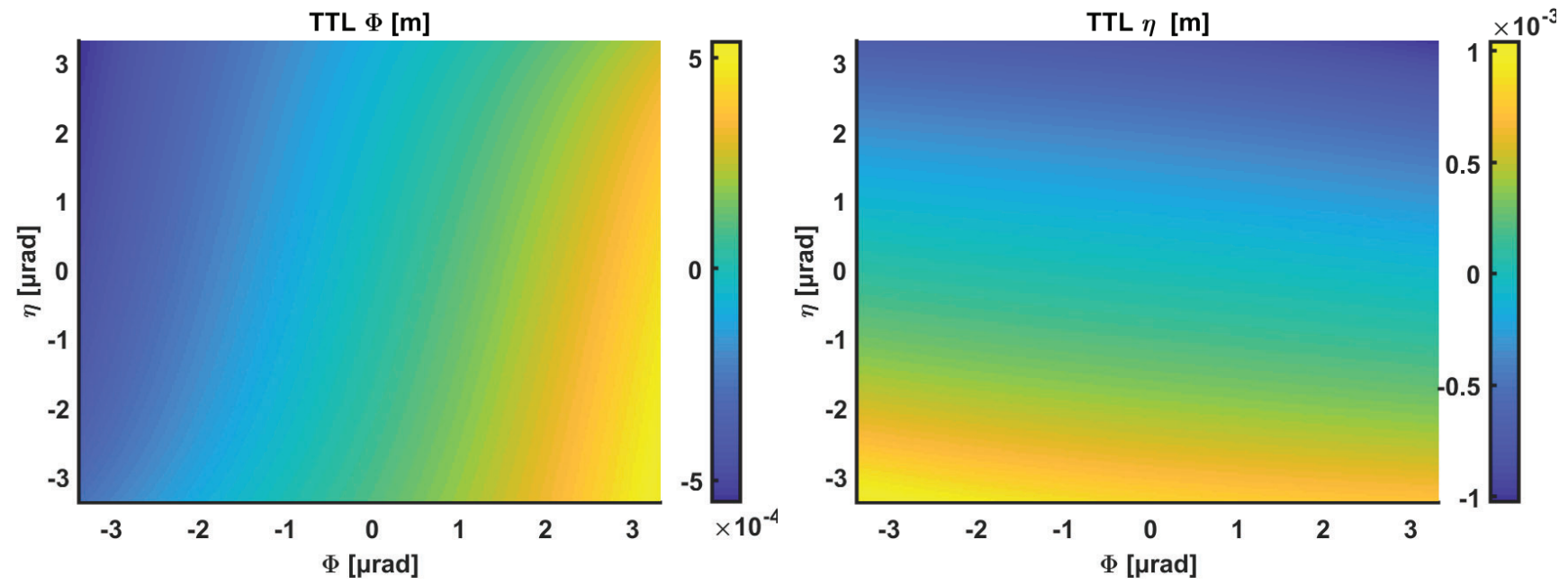

Figure 9: Far-field TTL coupling factors (i.e. angle derivative of far-field optical phase), as calculated from the measured WFE. Left: Coupling factor for angle $\Phi$, right: coupling factor for angle $\eta$. 
$500 \mathrm{nrad}$, the resulting maximum TTL coupling factor of $0.12 \mathrm{~mm}$ is well below the specification of $1 \mathrm{~mm}$ (which assumes a total pointing jitter of $10 \mathrm{nrad} / \sqrt{\mathrm{Hz}}$ ). However, it should be noted that archieving the same WFE for a LISA size telescope (typically twice the diameter) is not only more challenging, but also the coupling factor scales linearly with the diameter of the telescope.

\section{CONCLUSION}

In this proceeding, we detailed an alignment concept for a LISA representative telescope featuring a small, low mass actuated mirror positioned in an intermediate pupil plane for beam steering. The alignment procedure is based on measuring TTL effects to determine lateral offsets of the rotational axis of that mirror. Proceeding as presented here will provide information with respect to the minimal IFPM mirror piston achievable using such an alignment strategy. Furthermore, we described a concept for the experimental determination of telescope TTL - including the extraction or elimination of contributions due to mirror motion resulting from parasitic hinge deformation of the flexure hinges used in SCS and IFPM. While the RMS-wavefront error of the telescope $(\approx 0.035 \lambda)$ and computed far-field TTL coupling (Within the field of regard of $500 \mathrm{nrad}$, a maximum TTL coupling factor of $0.12 \mathrm{~mm}$ was computed) are within specification $(<1 \mathrm{~mm},<\lambda / 30)$, the angular jitter of the telescope is still roughly one order of magnitude larger than desired. Therefore, in order to obtain the specified pathlength stability, further measures have to be taken with respect to environmental stabilization. Currently, we are working on reducing angular noise by actively stabilizing the platform of the setup, thereby compensating seismic noise as well as supply pressure and CTE driven drifts of the pneumatic dampeners used for acoustic decoupling. As a final remark, it should be noted that in addition to TTL of pure geometric origin, also nongeometrical (e.g. wavefront related) TTL exists and plays an important role in real interferometers. ${ }^{67}$ These effects will also have to be considered when evaluating the measurement data.

\section{Acknowledgements}

Funding by the German BMWi via DLR is gratefully acknowledged as well as the ongoing fruitful cooperation with TNO.

\section{REFERENCES}

[1] Johann, U., Braxmaier, C., Holota, W., and Jörck, H., "Novel payload architectures for LISA," in [International Conference on Space Optics - ICSO 2006], 10567, 105670P, International Society for Optics and Photonics (2006).

[2] Danzmann, K., "Laser interferometer space antenna," arXiv:1702.00786 [astro-ph] (2017).

[3] Weise, D. R., Marenaci, P., Weimer, P., Schulte, H. R., Gath, P., and Johann, U., "Alternative optomechanical architectures for the LISA instrument," Journal of Physics: Conference Series 154, 012029 (2009).

[4] Brugger, C., Broll, B., Johann, U., Lucarelli, S., Voert, M., Weise, D., Fitzsimons, E., Jonke, W., Nikolov, S., and Witvoet, G., "An experiment to test in-field pointing for elisa," in [International Conference on Space Optics - ICSO 2014], 92 (2017).

[5] Witvoet, G., "Realization and performance validation of the in-field pointing mechanism for the evolved laser interferometer space antenna," ESA SP. , 737.10 (2015).

[6] Schuster, S., "Tilt-to-length coupling and diffraction aspects in satellite interferometry," (2017).

[7] Wanner, G., Heinzel, G., Kochkina, E., Mahrdt, C., Sheard, B. S., Schuster, S., and Danzmann, K., "Methods for simulating the readout of lengths and angles in laser interferometers with gaussian beams," Optics Communications 285(24), 4831-4839 (2012). 